ACCEPTED MANUSCRIPT

\title{
The stability of imaging biomarkers in radiomics: a framework for evaluation
}

To cite this article before publication: Helen Yu Chi Wang et al 2019 Phys. Med. Biol. in press https://doi.org/10.1088/1361-6560/ab23a7

\section{Manuscript version: Accepted Manuscript}

Accepted Manuscript is "the version of the article accepted for publication including all changes made as a result of the peer review process, and which may also include the addition to the article by IOP Publishing of a header, an article ID, a cover sheet and/or an 'Accepted

Manuscript' watermark, but excluding any other editing, typesetting or other changes made by IOP Publishing and/or its licensors"

This Accepted Manuscript is (C) 2018 Institute of Physics and Engineering in Medicine.

During the embargo period (the 12 month period from the publication of the Version of Record of this article), the Accepted Manuscript is fully protected by copyright and cannot be reused or reposted elsewhere.

As the Version of Record of this article is going to be / has been published on a subscription basis, this Accepted Manuscript is available for reuse under a CC BY-NC-ND 3.0 licence after the 12 month embargo period.

After the embargo period, everyone is permitted to use copy and redistribute this article for non-commercial purposes only, provided that they adhere to all the terms of the licence https://creativecommons.org/licences/by-nc-nd/3.0

Although reasonable endeavours have been taken to obtain all necessary permissions from third parties to include their copyrighted content within this article, their full citation and copyright line may not be present in this Accepted Manuscript version. Before using any content from this article, please refer to the Version of Record on IOPscience once published for full citation and copyright details, as permissions will likely be required. All third party content is fully copyright protected, unless specifically stated otherwise in the figure caption in the Version of Record.

View the article online for updates and enhancements. 


\title{
The stability of imaging biomarkers in radiomics: a framework for evaluation
}

\author{
HYC Wang ${ }^{1}$, EM Donovan ${ }^{1}$, A Nisbet ${ }^{2,3}$, CP South $^{2}$, S Alobaidli $^{1,4}$, V Ezhil ${ }^{5}$, I Phillips ${ }^{5}$, V \\ Prakash $^{5}$, M Ferreira ${ }^{6}$, P Webster $^{6}$, PM Evans ${ }^{1,3}$ \\ ${ }^{1}$ Centre for Vision, Speech and Signal Processing, University of Surrey, Guildford GU2 7XH, \\ UK \\ 2Department of Medical Physics, Royal Surrey County Hospital NHS Foundation Trust, \\ Guildford GU2 7XX, UK \\ ${ }^{3}$ National Physics Laboratory, Teddington, TW11 OLW, UK \\ ${ }^{4}$ Radiotherapy Physics, Kuwait Cancer Control Centre, Kuwait \\ ${ }^{5}$ Department of Oncology, Royal Surrey County Hospital NHS Foundation Trust, Guildford \\ GU2 7XX, UK \\ ${ }^{6}$ Alliance Medical Group, London SW1Y 6DN, UK \\ E-mail: h.y.wang@surrey.ac.uk
}

Keywords: radiomics, texture analysis, lung cancer, CT imaging

\section{Abstract}

This paper studies the sensitivity of a range of image texture parameters used in radiomics to: i) the number of intensity levels, ii) the method of quantisation to select the intensity levels and iii) the use of an intensity threshold. 43 commonly used texture features were studied for the gross target volume outlined on the CT component of PET/CT scans of 50 patients with non-small cell lung carcinoma (NSCLC). All cases were quantised for all values between 4 and 128 intensity levels using four commonly used quantisation methods. All results were analysed with and without a threshold range of $-200 \mathrm{HU}$ to $300 \mathrm{HU}$. Cases were ranked for each texture feature and for all quantisation methods with the Spearman's rank correlation coefficient determined to evaluate stability. Results showed large fluctuations in ranking, particularly for low numbers of levels, differences between quantisation methods and with the use of a threshold, with values Spearman's Rank Correlation for many parameters below 0.2. Our results demonstrated the sensitivity of radiomics features to the parameters used during analysis and highlight the risk of low reproducibility comparing studies with slightly different parameters. In terms of the lung cancer CT datasets, this study supports the use of 128 intensity levels, the same uniform quantiser applied to all scans and thresholding of the data. It also supports several of the features recommended in the literature for such studies such as skewness and kurtosis. A recommended framework is presented for curation of the data analysis process to ensure stability of results. 


\section{Introduction}

It is believed that standard-of-care medical images obtained from modalities such as CT, $\mathrm{MRI}$ and PET contain more information than is visible to the human eye (Lambin et al. 2012; Aerts et al. 2014). This underlying information may provide valuable additional, quantitative information about the subject. The emerging field of "radiomics" involves the high throughput extraction of quantitative imaging features with the intent of creating mineable databases from radiological images (Lambin et al. 2012). The additional quantitative data obtained could be used alongside the current dimensional measurements of tumour size e.g. Response Evaluation Criteria In Solid Tumours (1D) and World Health Organisation Longaxis (2D), as well as TNM staging information, for diagnosis, personalised treatment planning and prognosis for every patient (Jaffe et al. 2006). Lung cancer lends itself well to radiomics as it is central to both staging and treatment delivery, particularly radiotherapy. Lung cancer is the commonest cause of cancer mortality worldwide. This means any benefits from radiomics has the potential to have wide ranging effects (Alobaidli et al. 2014, Philips et al. 2018).

The features available for radiomics analysis are vast. Aerts et al. (Aerts et al. 2014) presented 440 features quantifying tumour image intensity, shape and texture. It is a common practice to build a machine learning model with all features, in order to find those which have prognostic power. This big data approach is prone to overfitting when used in medical imaging as the number of inputs i.e. the size of the cohort is often considerably smaller than the feature set.

Despite the underlying difference in the physics of acquisition of various imaging modalities, radiomics analysis contains four major processes with defined inputs and outputs: (a) image acquisition and reconstruction, (b) image segmentation and rendering, (c) feature extraction and feature quantification, (d) ad hoc informatics analyses (Kumar et al. 2012).

Many of the radiomics features not visible to the human eye are classified as texture. Texture describe the relationships between groups of voxels in an otherwise uniform region (such as in the tumour). Texture features may be classified in terms of the size of the pattern of voxels being studied. First order metrics are based on the histogram of the distribution of intensities, second order metrics analyse the relationships between pairs of voxels and higher order metrics analyse relationships between larger groups of voxels. The first stage of such analysis is to rescale (or re-quantise) the range of intensity, or grey, levels from the original image to produce a new number of intensity levels. This has the benefit of reducing noise.

Hence a key aspect of the process of texture analysis is quantisation. The segmented volume of the tissue being analysed needs first to be quantised so that information from the images can be extracted. A mathematical description of texture such as the grey-level cooccurrence matrix (GLCM) is generated from the quantised data. The matrix is then processed to generate measures such as entropy for texture quantification.

Quantisation is a vital step that groups image voxels with similar intensity level (or grey level) values to a smaller discrete set of values that are easier to manipulate. The size of the intensity set determines the size of the matrix built to calculate all texture parameters and hence determines the resolution of the features and computational power required. The majority of studies on texture analysis have used a uniform quantisation method with 16,32 , $64,128,256$ or 512 intensity levels often without investigating the effect of different numbers of levels (Yu et al. 2009; Vaidya et al. 2012; Fried et al. 2014; Leijenaar et al. 2015; Fave et al. 2015). The importance of quantisation and its impact on texture features has been overlooked compared to studies on the repeatability of texture features to changes in 
reconstruction settings and delineation (Galavis et al. 2010; Yan et al. 2015; Aerts et al. 2014; Altazi et al.2017).

Most quantisation methods proposed have used uniform quantisation to distribute linearly the raw image data into a set number of intensity levels (El Naqa et al. 2009; Tixier et al. 2011), or into levels with fixed bin width (Leijenaar et al. 2013; Leijenaar et al. 2015; Desseroit et al. 2017). When normalised, uniform quantisation methods allow direct comparison of features extracted between different images. An alternative approach is a clustering based method using the Lloyd-Max quantiser which allocates more intensity levels to the common input intensity values by minimizing the mean-squared quantisation error between input and output (Evans et al. 1997; South et al. 2009; Vallires et al. 2015). This is expected to retain more information than the uniform quantisation methods.

Desseroit et al. (Desseroit et al. 2017) compared the effectiveness of uniform quantisation with fixed intensity level number and fixed intensity bin width with PET and low-dose CT. Repeatability was tested for 4 shape descriptors, 10 first-order metrics and 26 texture features for 74 patients. The most reliable feature was found to be entropy calculated from the GLCM from PET quantised using 64 intensity levels followed by GLCM entropy from CT quantised using 10 Hounsfield units per bin, in agreement with earlier studies (Leijenaar et al. 2015; van Velden et al. 2016). Using fixed intensity level numbers with PET images produced more reliable features whereas features derived from CT images showed better results when quantised using fixed bin width.

The intensity range to be quantised lacks standardisation. CT scores intensity values as Hounsfield units (HU). Air, being the least attenuating, is defined as $-1000 \mathrm{HU}$, water has a $\mathrm{HU}$ of 0 . A few studies introduced a threshold on $\mathrm{HU}$ to reduce noise and focus the analysis on certain tissue such as tumour. Fave et al. 2014 and Fried et al. 2015 included regions with Hounsfield unit $-100 \mathrm{HU}$ to $200 \mathrm{HU}$, Ganeshan et al. 2010 excluded pixels below -50 HU, whereas others like Win 2013 did not use a threshold. A study performed by Mackin et al. 2015 to investigate inter-scanner variability measured a span of $-186 \mathrm{HU}$ to $35 \mathrm{HU}$ for NSCLC tumours. The impact of including a threshold was not investigated in any of the studies.

These studies illustrate that there are unanswered questions relating to the choice of parameters for texture analysis with potential consequences in terms of the stability of the results. In this paper stability is used to refer to the resulting metric not changing substantially with a small change in input parameters. A common use of these techniques is for stratifying patients (e.g. in terms of outcome) and hence stability is needed to maintain the ranking of radiomics features in a given dataset. If a slight change in the radiomics features input to machine learning algorithms causes a large change in output then stability is low and the results are likely to be meaningless and not reproducible. Whilst a wide variety of texture features have been reported as having prognostic value, often based on small single-centre patient cohorts, independent validation of these findings has proved problematic (e.g. McQuaid et al 2017). Instability of texture parameters to variations in input parameters and dichotomisation thresholds is likely to be a confounding factor.

Our investigation of the stability of texture features, commonly used in radiomics analysis, involved evaluating the effect of: i) the number of intensity levels used; ii) the quantisation method and iii) the application of an intensity level threshold.

In addition, we aimed to develop a methodology which would identify a set of stable features to be used as input to radiomics analysis. This study was carried out on a dataset of CT scans of 50 patients treated for lung cancer at The Royal Surrey County NHS Foundation Trust. 


\section{Methods}

This study evaluated the stability of commonly used texture features in radiomics by measuring their sensitivity to small changes in input parameters. This methodology was applied to CT scans of 50 NSCLC patients. The scans used were standard scans taken from PET/CT scanners. The PET/CT scans were used as these scans did not involve the use of contrast agent, which is routinely used for radiotherapy planning CT scans.

\subsection{Image Data}

CT image datasets from fifty NSCLC patients who underwent [18 ${ }^{\mathrm{F}}$ ]FDG PET/CT scans were available for analysis. The image datasets were obtained using four scanner types: Siemens Biograph 64 (26 patients); Siemens SOMATOM Definition AS (13 patients); GE LightSpeed RT16 (10 patients) and GE Discovery LS (1 patient) (Table 1). Each CT image size was 512 x 512 pixels, with pixel size $0.98 \mathrm{~mm} \times 0.98 \mathrm{~mm}$ and a slice thickness of $3 \mathrm{~mm}$ with both Siemens scanners and $2.5 \mathrm{~mm}$ using the GE scanners. Gross tumour volume (GTV) was outlined on the CT image sets by a clinical oncologist (IP, VE) or radiologist (VP) following a documented clinical protocol. The patients were selected sequentially from the Royal Surrey database. A range of scanner models was used as large radiomics studies are likely to require the use of multiple scanners. It was also instructive to know if any patterns found were present in different scanner models.

\subsection{Texture features}

The texture analysis toolkit of Vallires et al. (2015) ${ }^{1}$ was used to extract 43 standard features from the CT defined GTV. 3 were first order features and 40 were second or higher order of which 9 were from the GLCM, 13 from the Grey Level Run Length Matrix (GLRLM), 13 from the Grey Level Size Zone Matrix (GLSZM) and 5 from the Neighbourhood Grey Tone Difference Matrix (NGTDM) (Table 2). For the second and higher order features, matrices were generated to assess the relationship between the centre voxel and its neighbours. One matrix was generated for each of the 13 directions in 3-dimentional space, the texture features were calculated then averaged over the GTV volume.

\subsection{Parameters studied}

Stability was analysed by varying the parameters of the texture metrics and evaluating both their effect on the measured quantity and on the ranking of the dataset amongst the 50 cases. Parameters studied were: i) number of intensity levels, ii) method of quantisation and iii) the use of a threshold intensity. Variations were evaluated against a reference set of parameters of 128 intensity levels, the Group Uniform Quantiser (see below) and the use of a threshold. In addition the relationship between the ordering of the datasets and the volume of the GTV was studied, as a strong correlation with GTV volume would imply that a texture feature is a surrogate for tumour volume and hence adds no new information.

\subsubsection{Number of intensity levels}

All integer numbers of intensity level from 4 to 128 were studied to test the stability of each feature with intensity number changes. If changing the number of intensity levels e.g. from 55 to 54 or 56 produced a large change in a given feature this implies instability and that the number of intensity levels would be inappropriate to use in clinical studies. The range of 
intensity levels was chosen to include those reported in the literature including the conventional $2^{n}$ range (Yu et al. 2009; Vaidya et al. 2012; Fried et al. 2014; Leijenaar et al. 2015; Fave et al. 2015), 100 levels used by Vallieres et al.; Vallieres et al. 2017) and methods of fixing intensity bin width to $10 \mathrm{HU}$ (Desseroit et al. 2017) and $16 \mathrm{HU}$ (Fave et al. 2015).

\subsubsection{Quantisation methods}

Four quantisation methods encountered in the literature were used: three methods of uniform quantisation plus the optimised Lloyd-Max Quantiser. For an image I, let $I(x)$ represent the intensity value stored in voxel $x$, and let $N$ be the number of intensity levels.

(i) Individualised Uniform Quantisation (IUQ), where the range of intensities / of each individual image dataset is determined based on the individual maximum $\left(I_{\max }\right)$ and minimum $\left(I_{\min }\right)$ intensities in that dataset. Consequently each dataset will have a different quantisation. For voxel, $\mathrm{x}$ :

$$
I_{I U Q}(x)=\left[N \times\left(\frac{I(x)-I_{\min }}{I_{\max }-I_{\min }}\right)\right]
$$

(ii) Individualised Uniform Quantisation with Zero (IUQ0), where the range of intensities of each individual dataset is determined based on maximum intensity $\left(I_{\max }\right)$ for that dataset and the minimum intensity is zero for the density of air ( $H U$ of $\left.I_{\text {air }}=-1000\right)$

$$
I_{I U Q 0}(x)=\left[N \times\left(\frac{I(x)-I_{\text {air }}}{I_{\text {max }}-I_{\text {air }}}\right)\right]
$$

(iii) Group Uniform Quantisation (GUQ), where the range of intensities is the same for all datasets and based on the group maximum (Igroup-max) and group minimum (Igroup-min) intensities.

$$
I_{G U Q}(x)=\left[N \times\left(\frac{I(x)-I_{\text {group }-\min }}{I_{\text {group }- \text { max }}-I_{\text {group }-\min }}\right)\right]
$$

(iv) Optimised Lloyd Max Quantísation (LMQ), an iterative optimisation method that tests multiple combinations of transition and reconstruction levels to determine the optimal quantisation levels. The quantiser maps the continuous input variable range into a discrete set of levels to minimise the voxel-to-voxel mean square error between the quantised data and the original data. More detail may be found in South et al. (2009).

\subsubsection{Intensity range threshold}

An intensity window was defined and applied to the raw CT data before repeating the quantisation with the four methods using 4 to 128 intensity levels as previously. Voxels within the GTV containing an intensity value of $-200 \mathrm{HU}$ to $300 \mathrm{HU}$ ) were included in the analysis. The threshold was chosen to span the range found in the literature to be discriminative of lung nodules yet inclusive to small GTVs and early stage tumorous tissue (Fave et al. 2015; Fried et al. 2014; Ganeshan et al. 2010; Mackin et al. 2015). Results with the threshold were compared with unthresholded results.

\subsection{Correlation with volume}


The hypothesis that texture features contain information and are not just surrogates of tumour volume was tested by evaluating the correlation of each texture feature with GTV size.

\subsection{Stability analysis}

Stability was analysed by evaluating the variation in the texture metrics with changes in the parameters listed above. The Spearman's rank correlation was used to quantify the effect of changing each parameter on the rank order of the 50 patients' image sets for each texture quantity.

The Spearman's rank correlation was calculated using Equation 4, where $\operatorname{Cov}\left(r_{x}, r_{y}\right)$ is the Covariance of the rank of the two items of interest (the rank for one set of parameters and the rank after a small change in one of those parameters). $\sigma_{x}$ and $\sigma_{y}$ are the standard deviations for the two items of interest respectively.

$$
r_{s}=\frac{\operatorname{Cov}\left(r_{x}, r_{y}\right)}{\sigma_{x} \sigma_{y}}
$$

In addition to the stability analysis, the Spearman's rank correlation was used to evaluate the correlation of each texture feature with GTV volume, to establish if the feature is a surrogate for GTV and hence adds no extra information.

A good texture feature will have a high value of $r_{s}$ for changes in the input parameters but not too high a rank correlation with volume. For volume analysis, three subgroups were created: small (26 image sets) with GTV to $99 \mathrm{~cm}^{3}$; medium (17 image sets) with GTV size from $100 \mathrm{~cm}^{3}$ to $249 \mathrm{~cm}^{3}$ and large (7 image sets) with GTV greater than $250 \mathrm{~cm}^{3}$.

The literature was surveyed to identify texture features reported to be prognostic from studies using PET/CT imaging data of NSCLC patients. These features are discussed in the results. 


\section{Results}

Figures 1 and 2 show GLCM entropy for all 50 image data sets and for the 26 image data sets scanned on the Siemens Biograph 64, respectively. Data are shown for all quantisation methods and the use of thresholding. GLCM entropy is used as an example to demonstrate the common behaviour observed across the 43 features. Each line is an individual dataset; the line colour reflects the GTV size (black for small, blue for medium, red for large).

\subsection{Effect of scanner, number of intensity levels and quantisation method}

Comparison of Fig 1 and Fig 2 show very similar characteristic behaviour showing the features observed are a consequence of the texture feature analysis. Oscillations in the texture measure were observed across the entire spectrum of intensity levels for all four quantisation methods, without $(A-D)$ and with $(E-H)$ threshold. All plots display an oscillatorylike pattern between neighbouring numbers of intensity levels.

The fluctuation pattern is more apparent for the uniform quantisation methods (GUQ, IUQ, IUQ0) (panels A-C and E-G)) at low numbers of intensity levels indicating the potentially high risk of obtaining unreproducible results under these conditions. The magnitude of the fluctuations seen for the uniform quantisation methods decreased as number of intensity levels increases, becoming almost negligible from 64 levels. The pattern is different for LMQ $(\mathrm{D}$ and $\mathrm{H}$ ) where the magnitude of the oscillations is less across all numbers of intensity levels but remains for 64 or more levels.

All texture features vary less above 64 intensity levels, therefore for this data set quantising to 128 levels would provide a set of reproducible measures irrespective of quantisation method. For other datasets it would be instructive to carry out a similar analysis to ensure the texture measure is stable. The absolute value of all texture features increased with intensity level number and varied between the quantisation methods, with LMQ producing the highest absolute values but a narrower spread compared to the other quantisation methods indicating that combining data with different quantisation methods or numbers of levels is not to be advised.

\subsection{Effect of threshold}

The effects of threshold may be seen by comparing panels $A$ with $E, B$ with $F, C$ with $G$ and $\mathrm{D}$ with $\mathrm{H}$ in Fig 1. Refining the intensity range quantised using a threshold had the effect of reducing the severity of the high frequency fluctuations, producing stability for numbers of intensity levels as low as 16 using the IUQ, IUQ0 and LMQ methods. The smoothing effect was present but less prominent with GUQ. This implies that the application of a threshold (often chosen to exclude bone and air pockets) can result in improved stability of the texture feature values. The plot analysis method allows the observer to deduce what are stable quantiser and intensity level choices for a particular dataset. 


\section{GLCM.Entropy for All Cases}
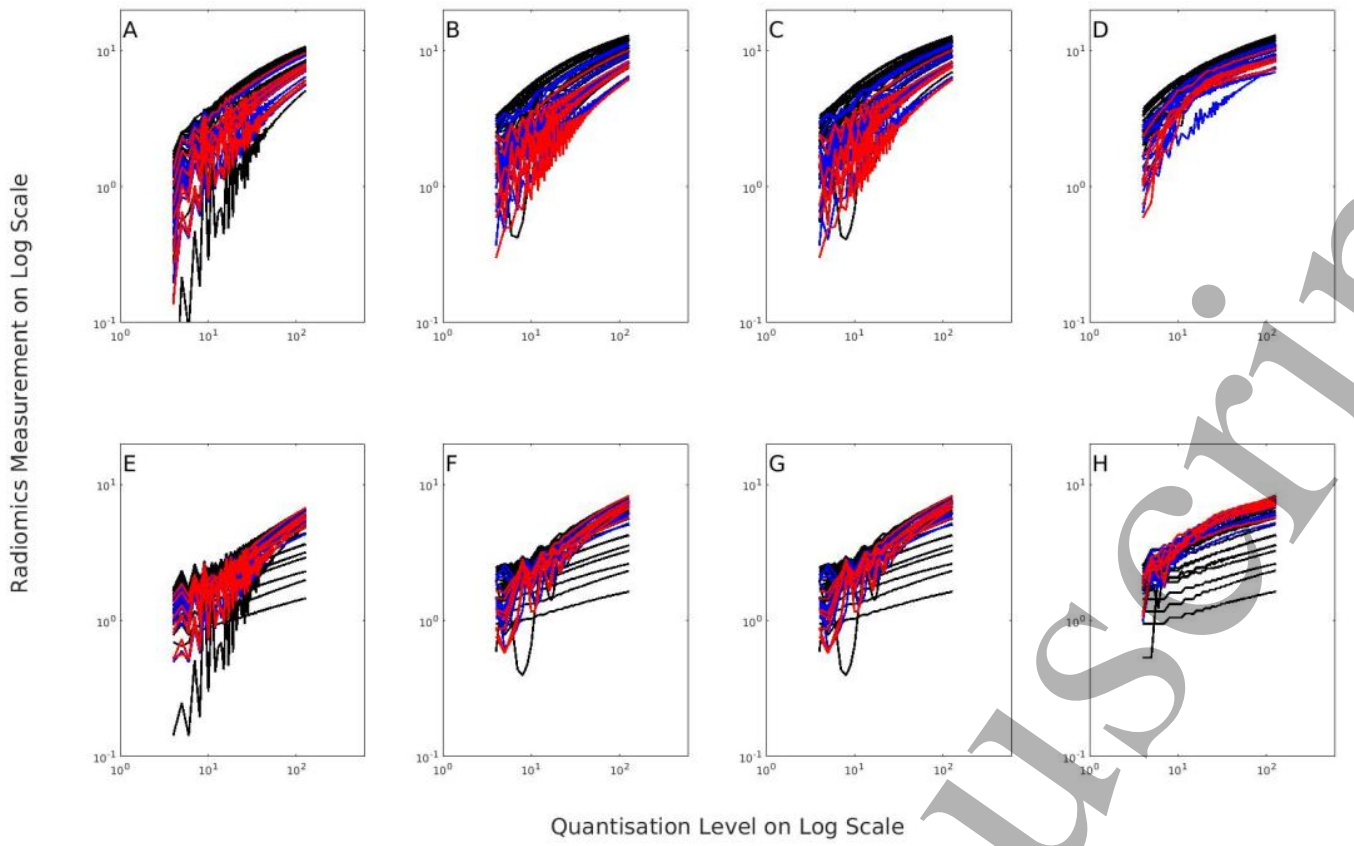

Quantisation Level on Log Scale

Figure 1: Plots of entropy as a function of number of intensity levels for all 50 patient $C T$ images. Panels $A$ and $E$ are for GUQ, $B$ and $F$ for IUQ, $C$ and $G$ for IUQO and $D$ and $H$ for LMQ. A-D are for unthresholded and $E-H$ for thresholded analysis. Black shows small GTV volume, blue medium, and red large

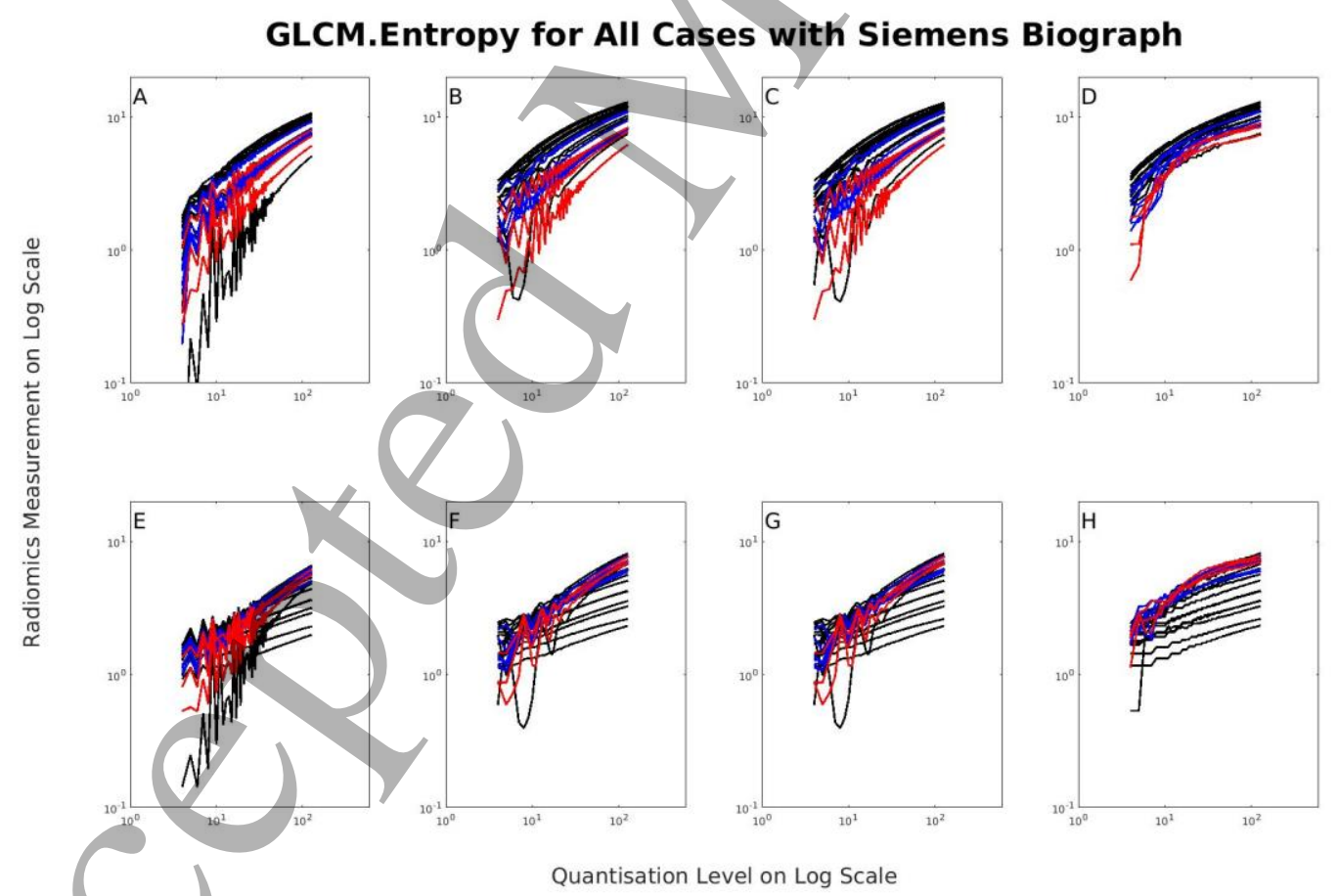

Figure 2: Plots of entropy as a function of number of intensity levels for 26 CT images from the Siemens Biograph scanner. Panels $A$ and $E$ are for GUQ, $B$ and $F$ for IUQ, $C$ and $G$ for IUQO and $D$ and $H$ for LMQ. A-D are for unthresholded and E-H for thresholded analysis. Black shows small GTV volume, blue medium, and red large 


\subsection{Correlation with volume}

Table 2 summarises the rank correlation coefficients of the 43 features with GTV size. A high correlation coefficient implies that the feature may be a surrogate for volume and hence may add no new information.

The effect of thresholding varied depending on GTV size. There was a greater reduction in the absolute value of texture features for the small GTV group compared to the medium and large groups. This behaviour is seen in Figure 1 with the black lines for the lower GTV volumes very different in the lower panels compared to the upper panels. This shows that analysing sensitivity to thresholding is particularly important for smaller tumour volumes.

\subsection{Stability analysis}

Figure 3 shows a plot of rank ordering for each of the 50 cases as a function of number of intensity levels, with the lowest value given rank 1 and the highest rank 50. The top panel shows entropy from GLCM and the bottom panel GLRLM LGHGE (Long Run High Greylevel Emphasis). In each, $A$ is unthresholded GUQ, $B$ is unthresholded $L M Q, C$ is thresholded GUQ and D is thresholded $L M Q$. For a stable radiomics metric the ordering should be independent of these parameters. As can be seen the order/changes with number of intensity levels particularly for low numbers. The Spearman's rank correlation describes this numerically. Figure 4 shows the Spearman's rank correlation as a function of number of intensity levels (compared to 128 levels) for each of the eight plots in Fig 3 . Fig 4 shows that for some combinations there is a lot of deviation in $r_{s}$ from the desired value of 1 . This is shown by GLCM entropy with thresholding for GUQ and LMQ (to a lesser extent), also for GLRLM LRHGE for the LMQ quantiser and a threshold. For the first case over 60 intensity levels are needed for the rank correlation with the highest level number to be above 0.9.

Table 2 lists Spearman's rank coefficient values for each of the 43 metrics. In this analysis, a reference set of parameters of 128 intensity levels, GUQ and thresholding is used. Then the effects of changing one of these parameters is measured in the Spearman's rank analysis to measure the stability of the rank order with a change of this parameter and hence the stability of the parameter.

In this table column 1 lists the metrics. Column 4 shows the rank correlation with volume. A high value of this would suggest surrogacy for volume. Values above 0.8 are shown in bold to indicate metrics which may be surrogates for volume. Column 5 shows rank stability for thresholding. Column 6 compares 16 with 128 intensity levels. Columns 7 to 9 compares GUQ with the other three quantisation methods. For all these columns a high rank correlation coefficient is desired to show a metric is stable to the choice of parameter. Values below 0.5 are shown in bold to indicate these metrics which may be unstable.

In the literature 23 features were reported to be prognostic: 16 for CT images; 13 for PET imaging with 6 prognostic with both. These are also shown in columns 2 and 3 in table 2 . Not all features reported to be prognostic in the literature had high Spearman's rank correlation in our analysis, nor were all stable features identified in this study reported to be prognostic in the literature.

Columns 9-12 show that many metrics have a high rank correlation for thresholded data, showing stability for different choices of quantisation method. For the unthresholded data the stability was generally lower, suggesting that the thresholding of data should be recommended. 


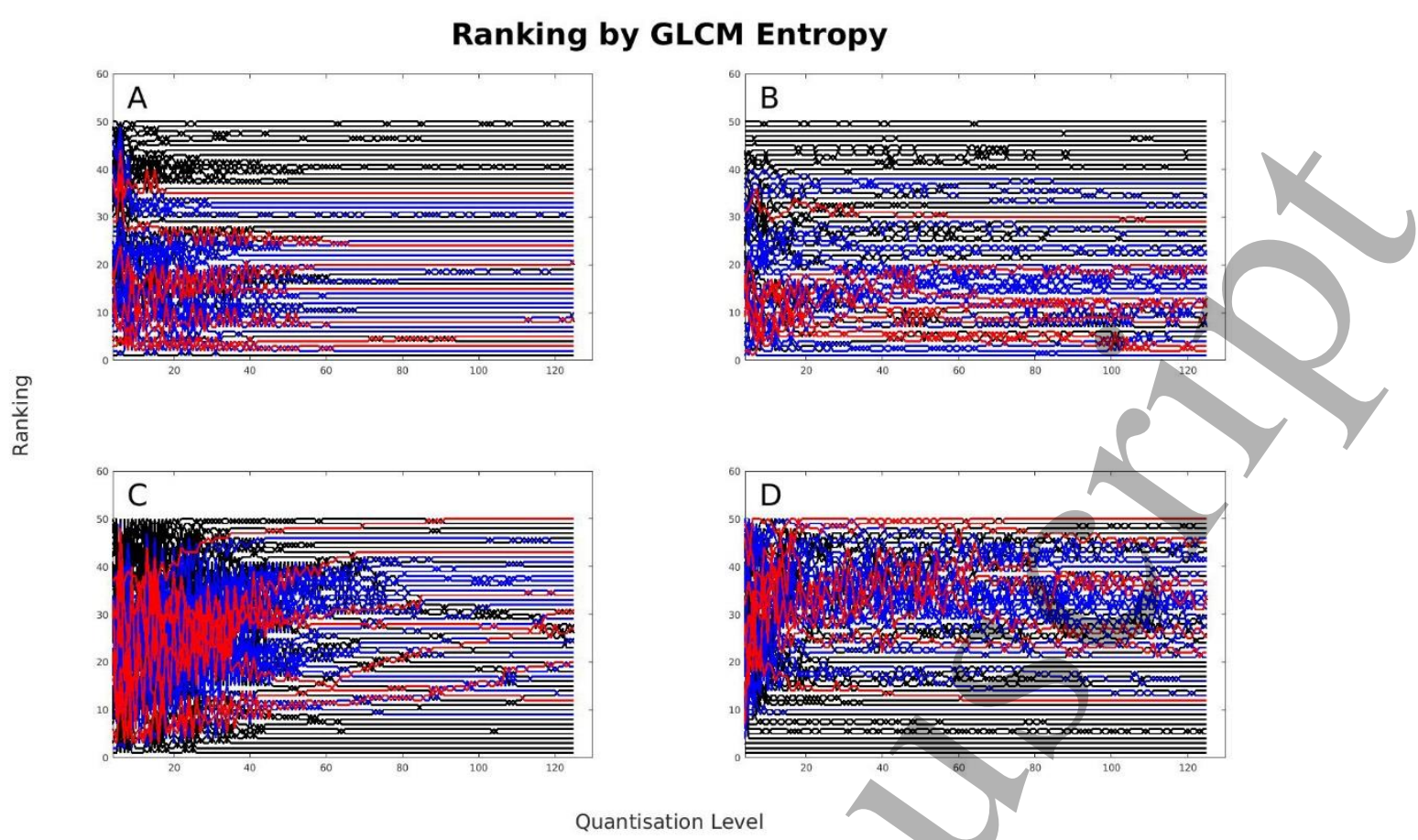

Ranking by GLRLM LRHGE

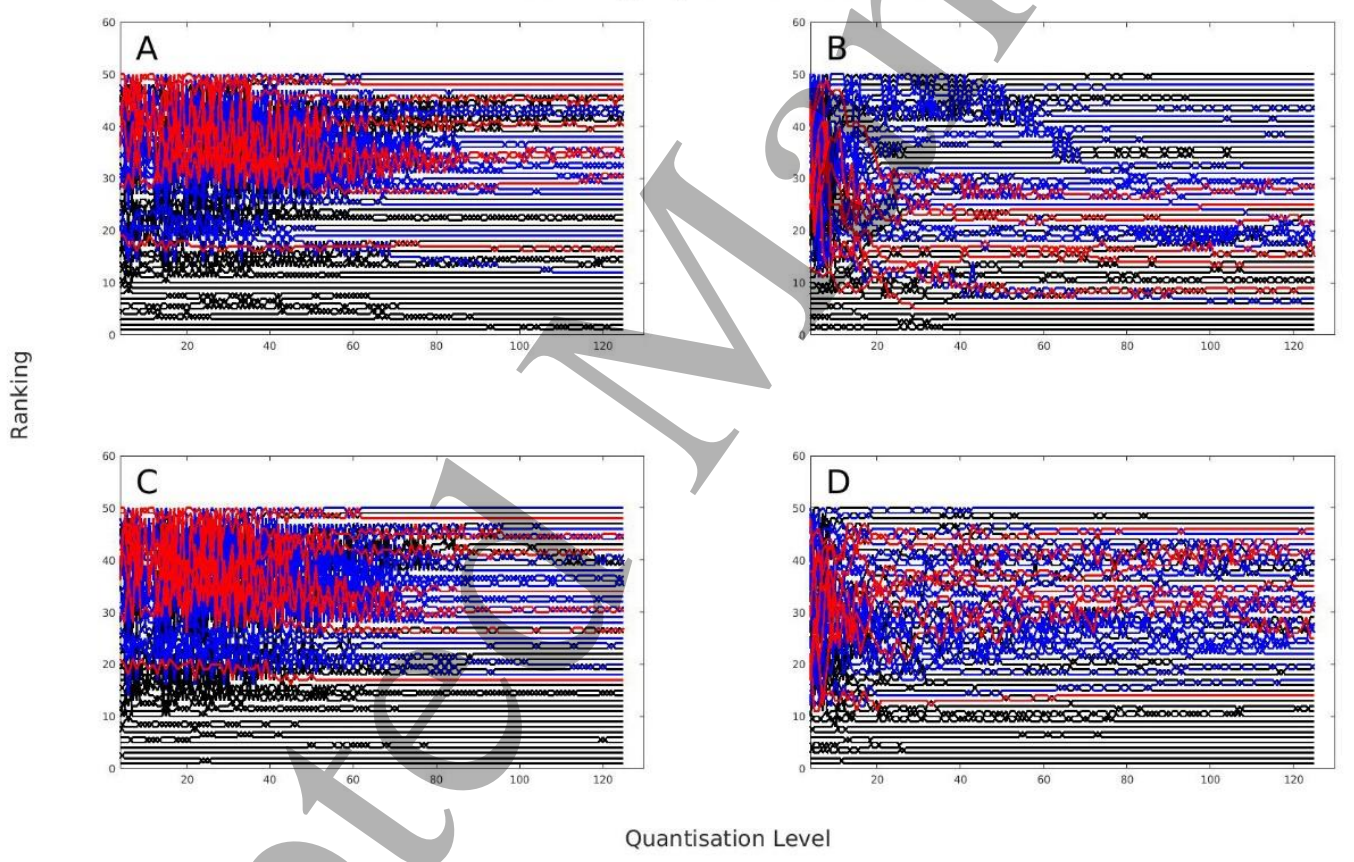

Figure 3: Ranking of datasets by (top) GLCM entropy and (bottom) GLRLM LRHGE for all numbers of intensity levels. Colour coded lines represent GTV size from smallest to largest: black, blue and red. $X$-axis is number of intensity levels. $Y$-axis is ranking. Subplots: $A$ : unthresholded GUQ, B: unthresholded $L M Q, C$ : thresholded GUQ, D: thresholded $L M Q$. 


\section{1}

\section{rs for GLCM Entropy}

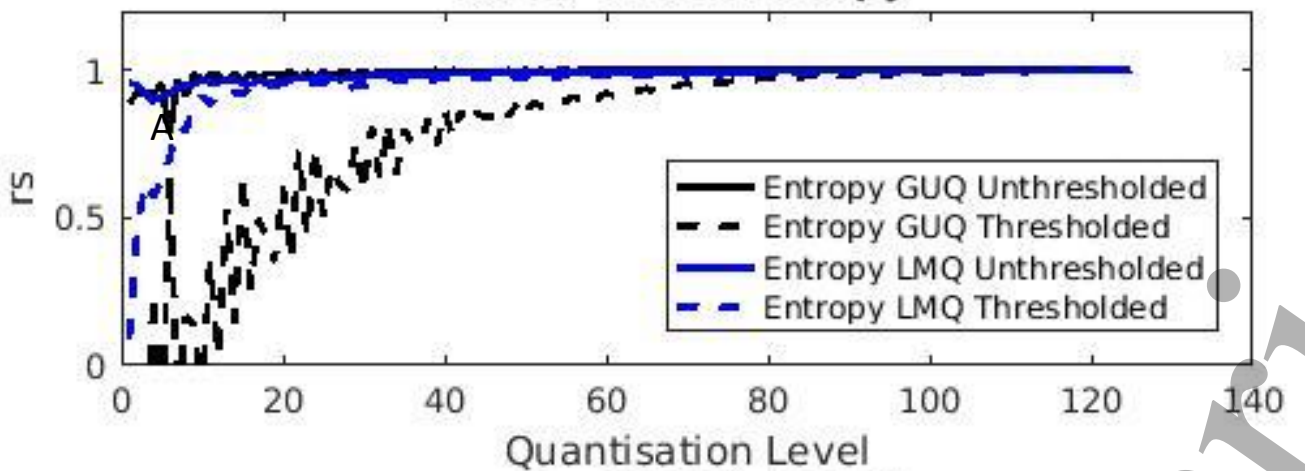

rs for GLRLM LRHGE

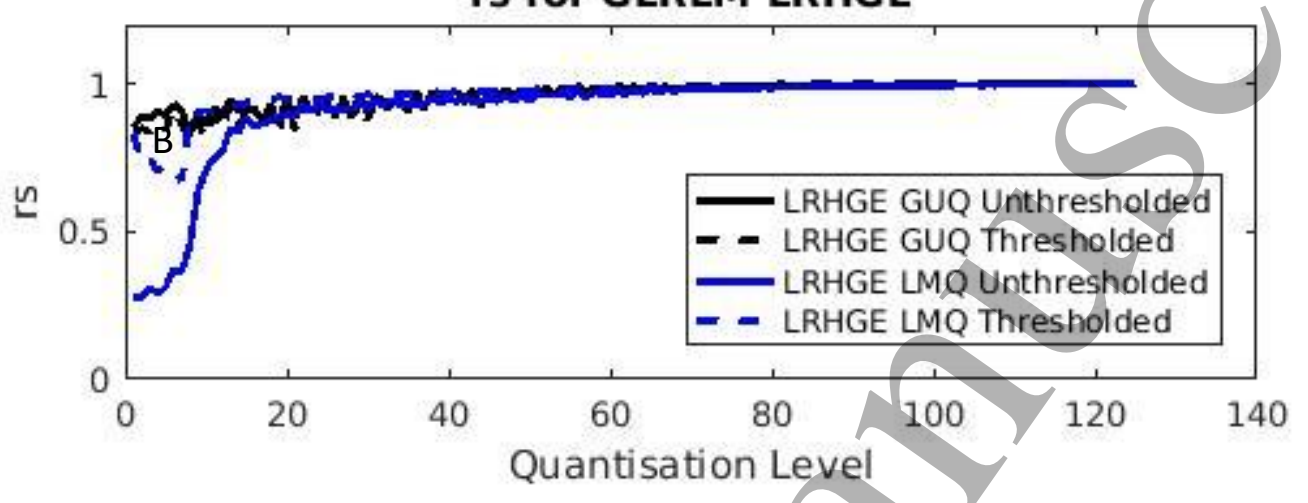

Figure 4: Value of the Spearman's rank coefficient, $r_{s}$, as a function of number of intensity levels. $r_{s}$ is plotted as the rank compared to 128 levels. A shows GLCM entropy and B GLRLM LRGHE for GUQ and LMQ quantisers and with and without the use of an intensity threshold. 


\section{Discussion and Conclusions}

This study evaluated the stability of commonly used features in radiomics. Stability was measured for i) variations in the number of intensity levels used, ii) the choice of method for selecting the intensity levels and iii) the use of a threshold intensity and was quantified using the Spearman's rank correlation. The rationale is that if a feature's value fluctuates by a large amount for a small change in input then it is not stable and hence unlikely to have predictive value. "A large amount" is interpreted as producing a substantial change in the rank order of the data set. Many radiomics studies involve using Kaplan-Meier analysis of outcome based on dichotomisation of datasets (e.g. Aerts et al. 2014, win et al. 2013). A change in rank order has the implication that a small change in parameters used to process the data could result in a data set moving from one dichotomised set to the other or oscillating between them as parameters are varied.

The risk of an unstable dataset may be ameliorated by carrying out an analysis to ensure that data and parameter choices are likely to produce stable results. The proposed framework is to use the methodology described in this work as a step between data acquisition and data analytics as shown in Figure 5.

The benefits of thresholding the data are highlighted in Table 2 where 10 of the 43 parameters show a rank correlation between GUQ and other quantisers below 0.5 without thresholding and only three are unstable with thresholding. Figures 3 and 4 show that small numbers of intensity levels are undesirable as they exhibit fluctuations in the value of the metrics GLCM Entropy and GLRLM LRHGE which is reflected in low Spearman's correlation. This might be interpreted as showing the need for the highest number of intensity levels possible, however this risks bringing noise in the images into the analysis (as small scale fluctuations in intensity), requires more processing power as histograms and matrices used in the generation of the texture features are larger and as panels A-D in Fig 1 show there is greater separation between the values of quantities such as entropy for midrange numbers of levels.

Two analysis decision values were illustrated: $r_{\mathrm{s}}<0.8$ for correlation with GTV volume to ensure the radiomics feature is not a surrogate for volume and $r_{s}>0.5$ for comparison of values with changes in number of intensity levels, quantisation method and threshold level, to ensure stability of the feature. These values are chosen as illustrations. In practice more work is needed to establish suitable values for both in a study.

In terms of the lung cancer CT datasets, this study supports the use of 128 intensity levels, GUQ and thresholding of data. Of the 43 features, 7 were found to correlate with volume, 20 were found to have poor stability with thresholding versus no threshold (this highlights the important of choosing a good threshold and large enough number of intensity levels) and only three were found to be sensitive to the choice of quantiser (providing a threshold was used).

In conclusion this paper has studied the stability of 43 commonly used radiomics features to: number of intensity levels, quantisation method and threshold for CT images of lung cancer patients, taken on PET/CT scanners. Stability of features is shown to be sensitive to all three parameters studied with optimal combinations providing greater stability. A recommended framework is presented for curation of the data analysis process to ensure stability of results. Such an approach could help to improve reproducibility of texture analysis across multiple sites and reduce false positives in radiomics studies. 
Study data acquisition

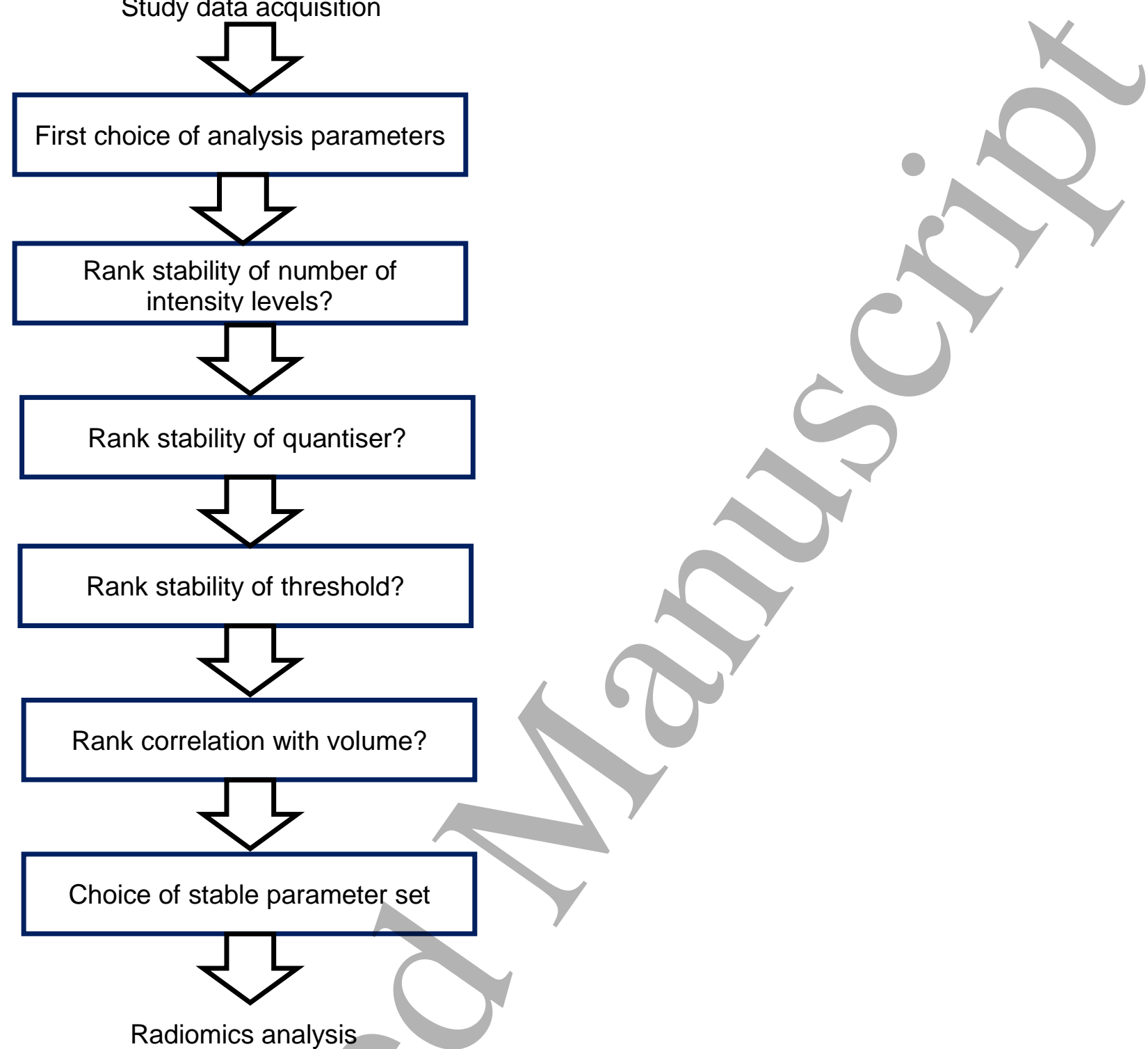

Radiomics analysis

Figure 5: Framework for stability analysis in radiomics

\section{Acknowledgement}

HYC Wang acknowledges PhD funding from Alliance Medical Limited. 


\section{References}

- Aerts, H.J., Velazquez, E.R., Leijenaar, R.T., Parmar, C., Grossmann, P., Carvalho, S., Bussink, J., Monshouwer, R., Haibe-Kains, B., Rietveld, D. and Hoebers, F., 2014. Decoding tumour phenotype by noninvasive imaging using a quantitative radiomics approach. Nature communications, 5, p.4006.

- Alobaidli, S., McQuaid, S., South, C., Prakash, V., Evans, P. and Nisbet, A., 2014. The role of texture analysis in imaging as an outcome predictor and potential tool in radiotherapy treatment planning. The British journal of radiology, 87(1042), p.20140369.

- Altazi, B.A., Zhang, G.G., Fernandez, D.C., Montejo, M.E., Hunt, D., Werner, J, Biagioli, M.C. and Moros, E.G., 2017. Reproducibility of F18-FDG PET radiomic features for different cervical tumor segmentation methods, gray-level discretization, and reconstruction algorithms. Journal of applied clinical medical physics, 18(6), pp.32-48.

- Brierley, J.D., Gospodarowicz, M.K. and Wittekind, C. eds., 2016. TNM classification of malignant tumours. John Wiley \& Sons.

- Desseroit, M.C., Tixier, F., Weber, W.A., Siegel, B.A., Le Rest, C.C., Visvikis, D. and Hatt, M., 2017. Reliability of PET/CT shape and heterogeneity features in functional and morphologic components of non-small cell lung cancer tumors: a repeatability analysis in a prospective multicenter cohort. Journal of Nuclear Medicine, 58(3), pp.406-411.

- Eisenhauer, E.A., Therasse, P., Bogaerts, J., Schwartz, L.H., Sargent, D., Ford, R., Dancey, J., Arbuck, S., Gwyther, S., Mooney, M. and Rubinstein, L., 2009. New response evaluation criteria in solid tumours: revised RECIST guideline (version 1.1). European journal of cancer, 45(2), pp.228-247.

- El Naqa, I., Grigsby, P.W., Apte, A., Kidd, E., Donnelly, E., Khullar, D., Chaudhari, S., Yang, D., Schmitt, M., Laforest, R. and Thorstad, W.L., 2009. Exploring feature-based approaches in PET images for predicting cancer treatment outcomes. Pattern recognition, 42(6), pp.11621171.

- Evans, P.M., Hansen, V.N. and Swindell, W., 1997. The optimum intensities for multiple static multileaf collimator field compensation. Medical physics, 24(7), pp.1147-1156.

- Fave, X., Mackin, D., Yang, J., Zhang, J., Fried, D., Balter, P., Followill, D., Gomez, D., Jones, A.K., Stingo, F. and Fontenot, J., 2015. Can radiomics features be reproducibly measured from CBCT images for patients with non-small cell lung cancer?. Medical physics, 42(12), pp.6784-6797.

- $\quad$ Fried, D.V., Tucker, S.L., Zhou, S., Liao, Z., Mawlawi, O., Ibbott, G. and Court, L.E., 2014. Prognostic value and reproducibility of pretreatment CT texture features in stage III non-small cell lung cancer. International Journal of Radiation Oncology* Biology* Physics, 90(4), pp.834-842.

- $\quad$ Frings, V., van Velden, F.H., Velasquez, L.M., Hayes, W., van de Ven, P.M., Hoekstra, O.S. and Boellaard, R., 2014. Repeatability of metabolically active tumor volume measurements with FDG PET/CT in advanced gastrointestinal malignancies: a multicenter study. Radiology, 273(2), pp.539-548.

- Galavis, P.E., Hollensen, C., Jallow, N., Paliwal, B. and Jeraj, R., 2010. Variability of textural features in FDG PET images due to different acquisition modes and reconstruction parameters. Acta Oncologica, 49(7), pp.1012-1016.

- Ganeshan, B., Miles, K.A., Babikir, S., Shortman, R., Afaq, A., Ardeshna, K.M., Groves, A.M. and Kayani, I., 2017. CT-based texture analysis potentially provides prognostic information omplementary to interim fdg-pet for patients with hodgkin's and aggressive non-hodgkin's lymphomas. European radiology, 27(3), pp.1012-1020.

- Ganeshan, B., Abaleke, S., Young, R.C., Chatwin, C.R. and Miles, K.A., 2010. Texture analysis of non-small cell lung cancer on unenhanced computed tomography: initial evidence for a relationship with tumour glucose metabolism and stage. Cancer imaging, 10(1), p.137.

- Goh, V., Sanghera, B., Wellsted, D.M., Sundin, J. and Halligan, S., 2009. Assessment of the spatial pattern of colorectal tumour perfusion estimated at perfusion CT using twodimensional fractal analysis. European radiology, 19(6), pp.1358-1365.

- Jaffe, C.C., 2006. Measures of response: RECIST, WHO, and new alternatives. Journal of Clinical Oncology, 24(20), pp.3245-3251. 
- Kumar, V., Gu, Y., Basu, S., Berglund, A., Eschrich, S.A., Schabath, M.B., Forster, K., Aerts, H.J., Dekker, A., Fenstermacher, D. and Goldgof, D.B., 2012. Radiomics: the process and the challenges. Magnetic resonance imaging, 30(9), pp.1234-1248.

- Lambin, P., Rios-Velazquez, E., Leijenaar, R., Carvalho, S., Van Stiphout, R.G., Granton, P., Zegers, C.M., Gillies, R., Boellard, R., Dekker, A. and Aerts, H.J., 2012. Radiomics: extracting more information from medical images using advanced feature analysis. European journal of cancer, 48(4), pp.441-446.

- Leijenaar, R.T., Carvalho, S., Velazquez, E.R., Van Elmpt, W.J., Parmar, C., Hoekstra, O.S. Hoekstra, C.J., Boellaard, R., Dekker, A.L., Gillies, R.J. and Aerts, H.J., 2013. Stability of FDG-PET Radiomics features: an integrated analysis of test-retest and inter-observer variability. Acta oncologica, 52(7), pp.1391-1397.

- Leijenaar, R.T., Nalbantov, G., Carvalho, S., Van Elmpt, W.J., Troost, E.G., Boellaard, R., Aerts, H.J., Gillies, R.J. and Lambin, P., 2015. The effect of SUV discretization in quantitative FDG-PET Radiomics: the need for standardized methodology in tumor texture analysis. Scientific reports, 5, p.11075.

- Mackin, D., Fave, X., Zhang, L., Fried, D., Yang, J., Taylor, B., Rodriguez-Rivera, E., Dodge, C., Jones, A.K. and Court, L., 2015. Measuring CT scanner variability of radiomics features. Investigative radiology, 50(11), p.757.

- McQuaid, S., Scuffham, J., Alobaidli, S., Prakash, V., Ezhil, V., Nisbet, A., South, C. and Evans, P., 2017. Factors influencing the robustness of P-value measurements in CT texture prognosis studies. Physics in Medicine \& Biology, 62(13), p.5403.

- $\quad$ Miller, A.B., Hoogstraten, B.F.A.U., Staquet, M.F.A.U. and Winkler, A., 1981. Reporting results of cancer treatment. cancer, 47(1), pp.207-214.

- $\quad$ Phillips, I., Ajaz, M., Ezhil, V., Prakash, V., Alobaidli, S., McQuaid, S.J., South, C., Scuffham, J., Nisbet, A., and Evans, P., 2018. Clinical applications of textural analysis in non-small cell lung cancer. The British Journal of Radiology, 91(1081), p.20170267.

- South, C.P., Evans, P.M. and Partridge, M., 2009. Dose prescription complexity versus tumor control probability in biologically conformal radiotherapy. Medical physics, 36(10), pp.43794388.

- $\quad$ Tixier, F., Le Rest, C.C., Hatt, M., Albarghach, N., Pradier, O., Metges, J.P., Corcos, L. and Visvikis, D., 2011. Intratumor heterogeneity characterized by textural features on baseline 18F-FDG PET images predicts response to concomitant radiochemotherapy in esophageal cancer. Journal of Nuclear Medicine, 52(3), pp.369-378.

- $\quad$ Vaidya, M., Creach, K.M., Frye, J., Dehdashti, F., Bradley, J.D. and El Naqa, I., 2012. Combined PET/CT image characteristics for radiotherapy tumor response in lung cancer. Radiotherapy and Oncology, 102(2), pp.239-245.

- $\quad$ Vallières, M., Freeman, C.R., Skamene, S.R. and El Naqa, I., 2015. A radiomics model from joint FDG-PET and MRI texture features for the prediction of lung metastases in soft-tissue sarcomas of the extremities. Physics in Medicine \& Biology, 60(14), p.5471.

- Vallières, M., Kay-Rivest, E., Rerrin, L.J., Liem, X., Furstoss, C., Aerts, H.J., Khaouam, N., Nguyen-Tan, P.F., Wang, C.S., Sultanem, K. and Seuntjens, J., 2017. Radiomics strategies for risk assessment of tumour failure in head-and-neck cancer. Scientific reports, 7(1), p.10117.

- $\quad$ van Velden, F.H., Kramer, G.M., Frings, V., Nissen, I.A., Mulder, E.R., de Langen, A.J., Hoekstra, O.S., Smit, E.F. and Boellaard, R., 2016. Repeatability of radiomic features in nonsmall-cell lung cancer [18 F] FDG-PET/CT studies: impact of reconstruction and delineation, Molecular imaging and biology, 18(5), pp.788-795.

- Win, T., Miles, K.A., Janes, S.M., Ganeshan, B., Shastry, M., Endozo, R., Meagher, M., Shortman, R.I., Wan, S., Kayani, I. and Ell, P.J., 2013. Tumor heterogeneity and permeability as measured on the CT component of PET/CT predict survival in patients with non-small cell lung cancer. Clinical Cancer Research, 19(13), pp.3591-3599.

- Yan, J., Chu-Shern, J.L., Loi, H.Y., Khor, L.K., Sinha, A.K., Quek, S.T., Tham, I.W. and Townsend, D., 2015. Impact of image reconstruction settings on texture features in 18F-FDG PET. Journal of nuclear medicine, 56(11), pp.1667-1673.

Yu, H., Caldwell, C., Mah, K., Poon, I., Balogh, J., MacKenzie, R., Khaouam, N. and Tirona, R., 2009. Automated radiation targeting in head-and-neck cancer using region-based texture analysis of PET and CT images. International Journal of Radiation Oncology* Biology* Physics, 75(2), pp.618-625. 


\begin{tabular}{|c|c|c|c|c|c|}
\hline $\begin{array}{l}\text { Patient } \\
\text { Number }\end{array}$ & $\begin{array}{l}\text { Tumour } \\
\text { Size }\left(\mathrm{cm}^{3}\right)\end{array}$ & $\begin{array}{l}\text { Manufacture } \\
\text { and Model }\end{array}$ & $\begin{array}{l}\text { Patient } \\
\text { Number }\end{array}$ & $\begin{array}{l}\text { Tumour } \\
\text { Size }\left(\mathrm{cm}^{3}\right)\end{array}$ & $\begin{array}{l}\text { Manufacture } \\
\text { and Model }\end{array}$ \\
\hline 1 & 3 & Siemens1 & 26 & 72 & Siemens2 \\
\hline 2 & 4 & Siemens2 & 27 & 108 & GE \\
\hline 3 & 5 & Siemens1 & 28 & 109 & Siemens1 \\
\hline 4 & 6 & Siemens2 & 29 & 112 & Siemens2 \\
\hline 5 & 6 & Siemens1 & 30 & 117 & Siemens1 \\
\hline 6 & 7 & Siemens1 & 31 & 118 & GE \\
\hline 7 & 10 & Siemens2 & 32 & 120 & Siemens1 \\
\hline 8 & 11 & Siemens1 & 33 & 140 & Siemens1 \\
\hline 9 & 21 & Siemens1 & 34 & 142 & $\mathrm{GE}$ \\
\hline 10 & 22 & Siemens1 & 35 & 146 & GE \\
\hline 11 & 27 & Siemens1 & 36 & 148 & Siemens1 \\
\hline 12 & 30 & Siemens1 & 37 & 168 & Siemens1 \\
\hline 13 & 30 & Siemens1 & 38 & 168 & $\mathrm{GE}$ \\
\hline 14 & 33 & GE & 39 & 181 & Siemens2 \\
\hline 15 & 35 & Siemens1 & 40 & 195 & Siemens1 \\
\hline 16 & 36 & GE & 41 & 200 & Siemens2 \\
\hline 17 & 36 & Siemens1 & 42 & 202 & Siemens2 \\
\hline 18 & 40 & Siemens1 & 43 & 217 & Siemens2 \\
\hline 19 & 41 & Siemens1 & 44 & 301 & Siemens2 \\
\hline 20 & 48 & Siemens1 & 45 & 356 & GELS \\
\hline 21 & 50 & $\mathrm{GE}$ & 46 & 419 & Siemens2 \\
\hline 22 & 53 & GE & 47 & 449 & Siemens2 \\
\hline 23 & 53 & Siemens1 & 48 & 513 & Siemens1 \\
\hline 24 & 61 & Siemens2 & 49 & 645 & Siemens1 \\
\hline 25 & 68 & GE & 50 & 988 & Siemens1 \\
\hline
\end{tabular}

Manufacturer and models are: Siemens1 - Siemens Biograph 64, Siemens2 - Siemens Somatom Definition AS, GE - GE Lightspeed RT16, GELS - GE Discovery LS

Table 1: List of patients by size of Gross Tumour Volume measured from the clinical outline data 


\begin{tabular}{|c|c|c|c|c|c|c|c|c|c|c|c|}
\hline \multirow[b]{2}{*}{ Feature name } & \multicolumn{2}{|c|}{ Prognostic in: } & \multirow{2}{*}{$\begin{array}{c}\text { Correlation } \\
\text { with volume }\end{array}$} & \multirow{2}{*}{$\begin{array}{c}\text { Thresholding } \\
\text { stability }\end{array}$} & \multirow{2}{*}{$\begin{array}{c}\text { Thresholded } \\
128 \mathrm{vs} 16\end{array}$} & \multicolumn{3}{|c|}{ Threshold GUQ vs: } & \multicolumn{3}{|c|}{ Unthreshold GUQ vs: } \\
\hline & $\mathrm{CT}$ & PET & & & & IUQ & IUQO & LMQ & IUQ & IUQO & LMQ \\
\hline Variance & & & 0.32 & 0.59 & 0.99 & 0.70 & 0.70 & 0.70 & 1.00 & 1.00 & 1.00 \\
\hline Skewness & 1 & 1 & 0.67 & 0.94 & 1.00 & 0.95 & 0.95 & 0.95 & 1.00 & 1.00 & 1.00 \\
\hline Kurtosis & 1 & 1 & 0.26 & 0.84 & 0.99 & 0.95 & 0.95 & 0.95 & 1.00 & 1.00 & 1.00 \\
\hline GLCM Energy & 1 & & 0.53 & -0.64 & 0.50 & 0.93 & 0.93 & 0.91 & 0.95 & 0.95 & 0.95 \\
\hline GLCM Contrast & 1 & 1 & 0.64 & 0.84 & 1.00 & 0.96 & 0.96 & 0.96 & 0.84 & 0.85 & 0.84 \\
\hline GLCM Entropy & 1 & 1 & 0.44 & -0.57 & 0.53 & 0.98 & 0.98 & 0.98 & 0.96 & 0.96 & 0.96 \\
\hline GLCM Homogeneity & 1 & & 0.36 & -0.50 & 0.45 & 0.97 & 0.97 & 0.97 & 0.94 & 0.94 & 0.94 \\
\hline GLCM Correlation & 1 & 1 & 0.47 & 0.87 & 1.00 & 1.00 & 1.00 & 1.00 & 1.00 & 1.00 & 0.88 \\
\hline GLCM SumAverage & & & 0.63 & 0.96 & 1.00 & 0.98 & 0.98 & 0.98 & 0.29 & 0.26 & 0.19 \\
\hline GLCM Variance & & & 0.25 & 0.80 & 0.99 & 0.94 & 0.94 & 0.94 & 0.84 & 0.86 & 0.83 \\
\hline GLCM Dissimilarity & 1 & & 0.62 & 0.80 & 0.98 & 0.99 & 0.99 & 0.99 & 0.93 & 0.93 & 0.92 \\
\hline GLCM Autocorrelation & & & 0.66 & 0.95 & 1.00 & 0.93 & 0.93 & 0.93 & 0.14 & 0.14 & 0.04 \\
\hline GLSZM SZE & & & 0.23 & 0.42 & 0.02 & 0.60 & 0.60 & 0.57 & 0.30 & 0.36 & 0.13 \\
\hline GLSZM LZE & 1 & & 0.83 & 0.65 & 0.93 & 0.91 & 0.91 & 0.89 & 0.98 & 0.99 & 0.97 \\
\hline GLSZM GLN & & & 0.41 & 0.36 & 0.39 & 0.23 & 0.23 & 0.22 & 0.39 & 0.43 & 0.36 \\
\hline GLSZM ZSN & & & 0.23 & 0.42 & 0.07 & 0.60 & 0.60 & 0.58 & 0.30 & 0.36 & 0.13 \\
\hline GLSZM ZP & & & 0.40 & 0.40 & 0.50 & 0.83 & 0.83 & 0.78 & 0.96 & 0.97 & 0.96 \\
\hline GLSZM LGZE & & 1 & 0.23 & -0.13 & 0.90 & 0.99 & 0.99 & 0.99 & 0.77 & 0.86 & 0.77 \\
\hline GLSZM HGZE & & 1 & 0.64 & 0.86 & 0.47 & 0.25 & 0.25 & 0.25 & 0.10 & 0.13 & 0.17 \\
\hline GLSZM SZLGE & & & 0.28 & -0.23 & 0.97 & 0.99 & 0.99 & 0.99 & 0.76 & 0.88 & 0.79 \\
\hline GLSZM SZHGE & & & 0.30 & 0.56 & 0.48 & 0.10 & 0.10 & 0.11 & 0.22 & 0.23 & 0.30 \\
\hline GLSZM LZLGE & & & 0.18 & -0.07 & 0.89 & 0.99 & 0.99 & 0.99 & 0.95 & 0.96 & 0.94 \\
\hline GLSZM LZHGE & 1 & & 0.93 & 0.99 & 0.98 & 0.99 & 0.99 & 0.99 & 0.99 & 1.00 & 0.98 \\
\hline GLRLM GLV & & & 0.92 & 0.90 & 0.93 & 0.97 & 0.97 & 0.97 & 0.97 & 0.97 & 0.94 \\
\hline GLSZM ZSV ) & 1 & & 0.89 & 0.46 & 0.90 & 0.96 & 0.96 & 0.96 & 0.83 & 0.85 & 0.79 \\
\hline GLRLM SRE & & 1 & 0.41 & -0.55 & 0.69 & 0.98 & 0.98 & 0.96 & 0.93 & 0.94 & 0.93 \\
\hline GLRLM LRE & & 1 & 0.41 & -0.60 & 0.17 & 0.97 & 0.97 & 0.96 & 0.91 & 0.92 & 0.91 \\
\hline GLRLM GLN & & 1 & 0.25 & -0.08 & 0.61 & 0.91 & 0.91 & 0.87 & 0.96 & 0.97 & 0.96 \\
\hline
\end{tabular}




\begin{tabular}{|c|c|c|c|c|c|c|c|c|c|c|}
\hline GLRLM RLN & & 0.41 & -0.56 & 0.63 & 0.98 & 0.98 & 0.96 & 0.94 & 0.94 & 0.93 \\
\hline GLRLM RP & & 0.45 & -0.61 & 0.33 & 0.98 & 0.98 & 0.97 & 0.92 & 0.92 & 0.92 \\
\hline GLRLM LGRE & 1 & 0.74 & 0.66 & 0.98 & 1.00 & 1.00 & 1.00 & 0.86 & 0.80 & 0.85 \\
\hline GLRLM HGRE & & 0.74 & 0.97 & 0.97 & 0.74 & 0.74 & 0.75 & 0.08 & 0.07 & 0.02 \\
\hline GLRLM SRLGE & & 0.83 & 0.45 & 0.88 & 1.00 & 1.00 & 1.00 & 0.86 & 0.81 & 0.85 \\
\hline GLRLM SRHGE & 1 & 0.67 & 0.96 & 0.88 & 0.61 & 0.61 & 0.64 & 0.00 & 0.00 & 0.03 \\
\hline GLRLM LRLGE & & 0.49 & 0.75 & 0.97 & 1.00 & 1.00 & 1.00 & 0.77 & 0.72 & 0.80 \\
\hline GLRLM LRHGE & 1 & 0.70 & 0.98 & 0.94 & 0.98 & 0.98 & 0.97 & 0.60 & 0.58 & 0.33 \\
\hline GLRLM GLV & & 0.34 & 0.82 & 0.60 & 0.97 & 0.97 & 0.97 & 0.68 & 0.67 & 0.66 \\
\hline GLRLM RLV & & 0.80 & 0.05 & 0.89 & 0.99 & 0.99 & 0.98 & 0.81 & 0.83 & 0.80 \\
\hline NGTDM Coarseness & & 0.78 & 0.72 & 0.91 & 0.98 & 0.98 & 0.98 & 1.00 & 1.00 & 0.98 \\
\hline NGTDM Contrast & & 0.58 & 0.89 & 0.95 & 0.99 & 0.99 & 0.99 & 0.98 & 0.99 & 0.98 \\
\hline NGTDM Busyness & 1 & 0.06 & 0.22 & 0.69 & 0.99 & 0.99 & 0.99 & 0.90 & 0.90 & 0.87 \\
\hline NGTDM Complexity & & 0.29 & 0.44 & 0.91 & 0.83 & 0.83 & 0.82 & 0.40 & 0.31 & 0.41 \\
\hline NGTDM Strength & & 0.96 & 0.72 & 0.97 & 1.00 & 1.00 & 1.00 & 0.83 & 0.83 & 0.75 \\
\hline
\end{tabular}

Table 2: Spearman's rank correlation coefficient for the 43 texture metrics. Columns 2 and 3 indicate if evidence for prognostic value has been found in the literature. Column 4 shows the correlation with volume, 5 shows the effects of thresholding, 6 compares 16 with 128 bins. The other columns 7-9 show the rank coefficient for different quantisers compared to GUQ for thresholded (7-9) and unthresholded (10-12) data. Bold data points indicate: 1) a correlation with volume above 0.8 in column 4, suggesting the metric may be a surrogate for volume and 2) a low rank correlation (below 0.5) for all other columns suggesting sensitivity to the choice of parameter and hence low stability. 